\title{
An Early Indication of the Rebound of W. Bancrofti Infection in Zanzibar
}

Shaali Ame ( $\square$ shaaliame@yahoo.com )

IVO

Dr Fatma M. Kabole

NTD Program, Ministry of Health Zanzibar

Salum Abuubakar

NTD Program, Ministry of Health Zanzibar

Dr Alphoncina Maseko Nanai

World Health Organisation

Dr Ghirmay Andemichael

World Health Organisation: Organisation mondiale de la Sante

Hugues Nana Djeunga

Centre for Research on Filariasis and other Tropical Diseases

\section{Research}

Keywords: Infection in Zanzibar, Lymphatic filariasis, ivermectin and albendazole

Posted Date: December 1st, 2020

DOI: https://doi.org/10.21203/rs.3.rs-114642/v1

License: (c) (i) This work is licensed under a Creative Commons Attribution 4.0 International License.

Read Full License 


\section{Abstract}

\section{Background}

Lymphatic filariasis (LF) is a debilitating neglected tropical disease which is targeted for elimination through co-administration of a single dose of ivermectin and albendazole in the affected population. Following implementation of such a treatment campaign over a period of years, control programmes are urged to conduct a transmission assessment survey to monitor the impact of the treatment and to ascertain whether the transmission is interrupted to a level that can no longer sustain transmission and hence mass drug administration (MDA) can be halted.

Objective:

This study was carried out to determine the prevalence of LF infection in Zanzibar communities, 13 years after stopping MDA, so as to inform and guide the control program.

\section{Methodology}

A Finger prick blood sample was collected from each participant after obtaining informed consent. The sample was assessed for the presence of Wucheria bancrofti circulating antigen using rapid immunochromatographic test.

Results

A total of 2555 subjects were enrolled (1231 in Pemba and 1324 in Unguja) in the study with a mean age of $23.0 \mathrm{yrs} \pm \mathrm{SD} 18.9(95 \% \mathrm{Cl}=22.3-23.8)$. There were more female $(53.9 \%)$ than male $(46.1 \%)$; and their mean age significantly differed (t-test $=7.5, p=0.00001)$. Only 2478 individuals gave blood sample. Of these, 88 (3.55\%) were found to be infected with $W$. bancrofti. Overall, the prevalence of infection was higher in Pemba (5.1\%) than in Unguja (2.1\%). The prevalence of infection was similar between different older age groups, children aged 1-5yrs in Pemba had the highest prevalence indicating that transmission is ongoing.

Observation of development of clinical manifestation, lymphoedema and hydrocele was also assessed. Overall, only $1.1 \%$ of the individuals had lymphoedema/elephantiasis; with male presented with more of those signs $(1.6 \%)$ than female $(0.7 \%)$. There was no male subject found to have hydrocele , although $8.3 \%$ of male had filariasis.

The assessment of treatment history revealed that majority (64.7\%) of the respondents had received at least one treatment round during their lifetime. Historical treatments with ivermectin did not correlate with current individual levels of infection but individuals who reported to have received 2 and 4 rounds of treatment were not found to be infected with filariasis. 
In view of our findings it clearly shows that the prevalence of LF in Zanzibar is still high to exceed the set threshold for discontinuation of MDA campaign. Children as young as 5yrs were found infected. It is therefore important to consider continuation of MDA so as to prevent potential disease sequel which might develop.

\section{Background}

The World Health Organisation (WHO) has set an ambitious goal of elimination of a number of neglected tropical diseases (NTDs) through a combination of strategies including mass drug administration (MDA), often recognised as preventive chemotherapy, in affected communities. MDA strategy has been demonstrated as an effective controlling measure for some of the NTDs, though its effectiveness depends on various factors such as a compliance and coverage (Smits, 2009).

Lymphatic filariasis (LF) is one of the NTDs targeted for elimination (World Health Organisation; Organization, 2010). During the global era of elimination of LF (Global Program to Elimination of Lymphatic Filariasis [GPELF]), preventive chemotherapy using a combination of oral single dose of ivermectin (IVM) and albendazole (ALB) was administered in communities across endemic areas (Ottesen, 2000). Such treatment campaigns eliminated or interrupted LF in most, if not all, countries after at least 5-6 rounds of its distribution (Rebollo et al., 2015b). Consequently, many endemic countries halted or scaled down distribution of ivermectin. Despite the earlier reports which suggested termination of drug distribution following 5-6 rounds (WHO, 2011), in fact the exact number of treatments to implement such decision was unclear. For example some endemic countries have implemented up to 14 rounds of MDA (Biritwum et al., 2019). The prematurely termination of drug distribution has in part, contributed to rebounce of infection in some endemic countries (Boyd et al., 2010).

Zanzibar has been long recognised as endemic for LF and so embarked in its elimination using the above-mentioned strategy similar like other LF endemic countries. Because of the stipulated number of treatment rounds (5-6) (Michael et al., 2006; Ottesen, 2000; Stolk et al., 2003), Zanzibar provided IVM and ALB for 6yrs from 2001-2006 (Mohammed et al., 2006). As a result, LF infection remarkably reduced to reach to the elimination threshold ( $<1 \%)$ (Mohammed et al., 2006). Following that achievement, again similar to other countries, Zanzibar stopped administration of IVM, and ALB directed towards LF. Ever since, there was no other intervention specifically targeted against LF, however, the continued efforts in malaria prevention/control measures and morbidity control for soil transmitted helminthiasis (STH) through provision of ALB, carried out in the Islands, indirectly benefited the LF control as demonstrated elsewhere (de Souza et al., 2015; Eigege et al., 2013; Kelly-Hope et al., 2013; Pion et al., 2017; Rebollo et al., 2015b) .

As part of the monitoring and evaluation, LF control programmes are urged to conduct transmission assessment survey (TAS), to understand if transmission has been completely interrupted and to demonstrate that there are no new LF cases in the communities. Zanzibar conducted its first, and indeed the only one to date, TAS in 2012 (Rebollo et al., 2015a). In that assessment the overall prevalence of 
infection was above 1\% (Rebollo et al., 2015a) and so warranted repeating of MDA with IVM. However, this was irregularly carried out between $(2013,2014$ and 2017). This present survey was conducted to assess the current status in terms of transmission of LF in communities on Pemba and Unguja islands to guide the NTD program on implementation of its control strategies and to understand if there is any early indication of a rebound of LF infection.

\section{Methodology}

\section{Selection of study sites.}

To ensure there is representation from each Island, the two Islands (Unguja and Pemba) were purposively selected, and each Island was considered as an independent evaluation unit (EU). The participated Shehias were selected based on the population size ( 500 individuals/Shehia as determined in 2012 national population census) and their previous history of high prevalence of infection observed in the TAS conducted in 2012 (Rebollo et al., 2015a). Efforts were made to ensure at least one site (shehia) was selected from each district. However, for various logistical reasons, that was not always possible.

Enrolment of participants and sample collection: The study field team in collaboration with shehia leaders, conducted a community mobilisation meeting and requested community members to turn-up to the designated sample collection area. The interested individuals were asked their consent for participation. The consent was administered orally. For participants $<18 y r s$, parents/guardian were asked to consent on their behalves. Attempts were made to ensure reasonable number of males and females of all age groups are enrolled. From each participant, a finger prick blood sample was collected and immediately analysed for the presence of Wuchereria bancrofti antigen using a rapid immunochromatographic test (ICT), Filariasis Test Strip (Alere ${ }^{\mathrm{TM}}$, (Alere Scarborough, Inc)). The test was performed as per manufacturer's instruction. Briefly, a finger prick was made and a whole blood sample was drawn using a capillary micropipette provided in the test kit. The sample was placed on the sample window and left uninterrupted at room temperature (RT) for 10 minutes. Thereafter, the test result was recorded. The sample was considered positive if visible purple lines were observed at the control and test windows. The sample was considered negative if only the control purple line was observed.

Once the blood sample was collected, each participant was requested to enter an examination room where physical examination and questionnaire were administered. Specifically, the questionnaire was meant to collect demographic information, treatment history, residency and travel history.

\section{Data management and analysis}

Data were double entered using an Epidata ver...the data were then cleaned and imported to excel and later imported to Stata version 13 (StataCorp, College Station) for analysis. The Chi Square test (X2) was used to assess association of categorical data and the probability of $\leq 0.05$ was considered as significant. 


\section{Results}

During the month of March 2019, a survey to investigate the prevalence of filariasis following intermittent provision of treatment in communities was carried out in 8 Shehias in both Unguja and Pemba (Table 1). A total of 2558 subjects were enrolled in the study. Although, for some reasons, the demography of some of the subjects was not recorded. Nevertheless, the participants had a mean age (23.0 yrs. \pm SD 18.9 [95\% $\mathrm{Cl}=22.3-23.8])$. There was slightly higher proportion of female (53.9\%) than male (46.1\%) enrolled in the study. Moreover, the mean age between the sexes significantly differed (25.6yrs vs. 20 for female and male, respectively) (t-test $=7.5 ; p=0.00001$ ).

Table 1: List of Shehia participated in the filariasis prevalence survey

\begin{tabular}{clcll} 
S/no. & Shehia name & No. of subjects & District & Island \\
\hline 1. & Finya & 294 & Wete & Pemba \\
2. & Maziwa Ng'ombe & 309 & Micheweni & Pemba \\
3. & Mkokotoni & 353 & Kaskazini A & Unguja \\
4. & Mzuri & 307 & Kusini & Unguja \\
5. & Ngwachani & 303 & Mkoani & Pemba \\
6. & Nungwi & 324 & Kaskazini A & Pemba \\
7. & Ole & 325 & Chake-chake & Pemba \\
8. & Pangawe & 340 & Magharibi B & Unguja \\
\hline
\end{tabular}

Unfortunately, not all the enrolled subjects gave blood sample for testing. Thus for determination of the prevalence of infection, only 2478 subjects were considered. Overall, 88 (3.55\%) individuals were infected with $W$. bancrofti as determined by the detection of filarial antigen. Interestingly there was no association of infection between sexes (3.5 vs. $3.6 \%$ for female and male respectively) ; $p=0.8$.

The analysis of filariasis infection in Shehias revealed a varying prevalence; with Ole having by far the highest $(12.1 \%)$ prevalence and Mkokotoni $(0.0 \%)$ had the lowest prevalence (Figure 1$)$, though, most of the surveyed Shehias had evidence of filariasis. The proportion of the infected individuals observed in Ole alone, equates to $44.3 \%$ of the combined infection across the whole study.

With regards to the prevalence of infection across the Islands, it showed that Pemba had higher prevalence $(5.1 \%)$ than Unguja (2.1\%).

\section{Prevalence of filaria infection in different age groups.}

The prevalence of filariasis infection in different age groups is shown in Figure 2. Generally, the prevalence of infection was similar between the different age groups, although younger children (aged between 1-5yrs) had a slightly higher infection rate. 
To further analyse the data across different age groups and between Pemba and Unguja islands, the figure showed a contrasting pattern (Figure 3). For Pemba, the pattern of filariasis was somewhat inconsistent in children (1-14yrs), though was more prominent in very young age group (1-5yrs). For the adult age groups (15 yrs), the infection steadily increased. Whilst for Unguja, there was more consistent pattern and the prevalence steadily increased with age group with highest infection rate detected at age group of 20-25yrs.

\section{Assessment of clinical manifestations resulting from filariasis}

In addition to collection of blood samples, development of clinical manifestation resulting from filariasis namely lymphoedema or elephantiasis and hydrocele were also observed. As our survey was community based, in some areas the environment was non-conducive to conduct thorough physical examination thus not all the subjects were ready to be examined for the presence of clinical manifestations especially hydrocele. Only 338 male subjects agreed for that. Of these, none had hydrocele, although $8.3 \%$ were found to be positive for filarial antigen; indicating presence of filariasis infection.

Similarly, for observation of lymphoedema or elephantiasis, only 936 subjects were concerned. Of these, overall, $1.1 \%$ (10) were found to have lymphoedema/elephantiasis. Interestingly there were more male subjects $(1.6 \%)$ who had lymphoedema/elephantiasis than female $(0.7 \%)$. Albeit, the presence of

lymphoedema/elephantiasis was statistically not associated with $\operatorname{sex}\left(\chi^{2}=1.6, p=0.2\right)$.

\section{Assessment of Treatment history}

We have assessed treatment history based on mass drug administration (MDA) directed towards control of filariasis (for the subjects) to understand how frequency of treatment could have impacted transmission of filariasis infection in communities. Individuals aged $<=7 y$ rs were not considered for this assessment as they were ineligible when they were $<5 y$ rs and also there was no treatment campaign in 2016 and 2017 (by this time those children could have turned to 7yrs). A total of 1199 individuals responded to the question. Of these $64.7 \%, 11.2 \%, 11.2 \%$ and $12.9 \%$ had received 1 (one), 2,3 and 4 rounds of treatment, respectively. Further analysis showed an inconsistent trend by observing infection (3.0\% and 5.3\%) in individuals who reported receiving, respectively 1 and 3 treatment rounds. All individuals who reported to receive 2 and 4 rounds were not found to be infected with filariasis.

\section{Discussion}

The suffering and debilitating effect of LF is well appreciated and some initiatives have been put in place to alleviate those suffering. Indeed in responding to the outcomes of infection, the WHO has endorsed resolution WHA 50.29 to urge endemic countries to eliminate LF as a public health problem (Addiss, 2013; Molyneux, 2003) and also focus on management of clinical conditions. The potential for elimination of the infection was boosted following advancement in disease diagnosis and the availability of the potent antimicrofilarial drug, ivermectin (Ottesen, 2000). It was demonstrated that regular distribution of a single 
oral dose of ivermectin over a period of years could successful interrupt transmission of LF and possibly eliminate the infection.

We report here the finding of the assessment of LF transmission in communities which have inconsistently received ivermectin treatment after stoppage in 2006. This survey followed a similar one conducted in 2012 (Rebollo et al., 2015a). In this present study an overall prevalence of $3.55 \%$ was demonstrated, though, varied between the communities (Fig.1). Interestingly the prevalence of infection was very similar between male (3.6\%) and female (3.5\%) and so the infection was not associated with sexes. Generally the proportion of infection was higher in Pemba (5.1\%) than in Unguja (2.1\%). Moreover, the prevalence of infection across the different age groups was similar, although a much higher in very young children (1-5yrs). When the data were further stratified in relation to age-groups and islands, overall, in Pemba, the prevalence was more prominent in younger children (1-5yrs) but there was inconsistent pattern for other age groups. For Unguja the prevalence of infection steadily increased with age groups with highest infection rate detected in adult age groups. Specifically, a steadily increase of infection rate was found in individuals aged 15yrs. Our finding of overall prevalence of $3.55 \%$ is slightly higher than what has been detected previously in the same setting (Rebollo et al., 2015a). The reason for observation of an overall increase of and/or decrease of infection between the two Islands has not been immediately established as there has no interventions carried out in all Islands specifically directed towards LF, other than side benefit which could result from malaria control initiatives and STH infectionsthrough provision of ALB. All environmental factors including rainfall patterns between the Islands remained almost constant. Nevertheless, it should be noted that in the earlier study (Rebollo et al., 2015a), only schoolchildren aged between 6-7yrs were investigated, as carried out in other endemic countries (Dewi et al., 2015). Whilst the present study investigated community members with a wide age range (286yrs). There are mixed perceptions on whether conducting a school-based or community-based TAS but other authors have drawn attention on possibility of leaving out non-school attendants some of whom might have not received the drugs (Biritwum et al., 2019). For examples in American Samoa, high prevalence of filariasis was observed in community-based assessment (6.2\%) than in school-based survey $(0.7 \%)$ (Sheel et al., 2018). Of note, despite variation of number of subjects and the age range between those two studies, the actual number of infected individuals was very similar (89 (2012) vs. 88(2019)). As aforementioned, the present study has observed LF infection in wide range of age groups, though slightly higher in children 1-5yrs. This finding is very alarming as these young children haven't been treated with ivermectin and indicates active transmission of infection in communities. Though studies showed that in LF endemic areas children become infected at very early age (Witt and Ottesen, 2001).

With regards to observation of clinical conditions, our study has demonstrated that none of the male subjects (agreed for physical examination) had hydrocele, albeit $8.3 \%$ of them were found to have filariasis. Similarly, only small proportion (1.1\%) of individuals were found to have lymphoedema/elephantiasis. The finding of the absence of development of hydrocele or low proportion of individuals with lymphoedema or elephantiasis could be possibly due to low parasite density or an acquired immunity, though microfilaria density was not established in this present study. There are 
limited studies investigated relationship of development of filariasis associated morbidity and worm burden but the existing evidence and experience from other nematodes infections indicates strong association between worm burden and progression to chronic clinical manifestations (Michael et al., 1994). Nevertheless, it should be noted that progression of those chronic stage of infection especially lymphoedema is a slow process which mostly occurs in older age and rarely found in individuals aged 20yrs (Chan et al., 1998). This observation might true, as in this present study, many of the infected individuals (66 [3.5\%]) were aged yrs (this analysis was not shown). Moreover, a longitudinal study conducted in Tanzania to assess development of hydrocele and lymphoedema revealed no association of the presence of microfilaremia and development of hydrocele or lymphoedema (Meyrowitsch et al., 1995).

The present study also assessed treatment history relative to LF based MDA in the population. Generally most individuals had received at least one round of IVM - MDA at certain point of time, however, many of them don't receive subsequent treatments. In this present study, it revealed that only $12.9 \%$ of the respondents received 4 rounds of IVM-MDA, a minimum number of treatment rounds that could achieve elimination. Surprisingly, an inconsistent trend of prevalence of infection was observed in relation to treatment history. Specifically, we have found substantial proportions (3.0\% and $5.3 \%$ ) of infection in individuals received one or three rounds of treatment respectively but not in individuals received 2 or 4 rounds of treatment. As hypothesized that, for LF based MDA at least 5 rounds of treatment (Ottesen, 2000 ) with reasonably coverage ( 65\%) (Stolk et al., 2003 ) are required to successfully interrupt transmission and eventual eliminate the disease. It is sensible to believe that our finding of the LF infection among previously treated individuals could not be a surprising phenomenon for many reasons including (i) many individuals were not constantly received repeated doses (sub-optimal treatment) of the drugs and that possibly had residual microfilaremia (ii) there was low drug coverage and so some members of the communities were in fact not treated- who were possibly infected and served as "reservoir"- although in reality, humans are not considered as reservoir of LF infection, and (iii) possibility of treatment failure. Although, the former might may play a little role as treatment failure has only been reported (Mahoney and Kessel, 1971) with diethylcarbamazine (DEC), another drug used in MDA to combat LF, but not with IVM.

In view of our findings it clearly shows that the prevalence of LF in Zanzibar is still high to exceed the set threshold for discontinuation of MDA campaign. Children as young as $5 y$ rs were found infected. It is therefore important to consider continuation of MDA so as to prevent potential disease sequel which might develop.

\section{Declarations}

\section{Ethics statement}

The ethical approval was obtained from the Zanzibar Medical Research and Ethics Committee (ZAMREC, reference no. ZAMREC 0003/Nov/2018) in Zanzibar, United Republic of Tanzania. The study participants 
were informed about the study objectives and procedures. Written consent was obtained from parents prior to sample collection from children. Participation was voluntary and participants could withdraw or be withdrawn.

The consent to public this work was also granted by the ZAMREC.

Conflict of interest: The authors declared no conflict of interest exists .

This study was funded by the World Health Organisation.

Conflict of interest: The authors declared no conflict of interest exists

Authors contributions

- SA: conceptualisation, design of the data collection tools, supervised field work analysed data and prepared manuscript

- FK: Conceptualisation, design of the data collection tools, supervised field work and prepared manuscript

- Sab: Conceptualisation, design of the data collection tools, supervised field work and prepared manuscript

- AN: prepared manuscript

- GA: prepared manuscript

- NG: Conceptualisation, participated in the design of the data collection tools

Acknowledgement

We would like to acknowledge:

- local leaders for their cooperation and engagement.

- Study participants

- all NTD program staff

- WHO for funding the study

\section{References}

Addiss, D.G., 2013. Global elimination of lymphatic filariasis: a "mass uprising of compassion." PLoS Negl. Trop. Dis. 7.

Biritwum, N.K., Frempong, K.K., Verver, S., Odoom, S., Alomatu, B., Asiedu, O., Kontoroupis, P., Yeboah, A., Hervie, E.T., Marfo, B., 2019. Progress towards lymphatic filariasis elimination in Ghana from 2000-2016: Analysis of microfilaria prevalence data from 430 communities. PLoS Negl. Trop. Dis. 13, e0007115. 
Boyd, A., Won, K.Y., McClintock, S.K., Donovan, C. V, Laney, S.J., Williams, S.A., Pilotte, N., Streit, T.G., de Rochars, M.V.E.B., Lammie, P.J., 2010. A community-based study of factors associated with continuing transmission of lymphatic filariasis in Leogane, Haiti. PLoS Negl. Trop. Dis. 4.

Chan, M.-S., Srividya, A., Norman, R.A., Pani, S.P., Ramaiah, K.D., Vanamail, P., Michael, E., Das, P.K., Bundy, D.A., 1998. Epifil: a dynamic model of infection and disease in lymphatic filariasis. Am. J. Trop. Med. Hyg. $59,606-614$.

de Souza, D.K., Ansumana, R., Sessay, S., Conteh, A., Koudou, B., Rebollo, M.P., Koroma, J., Boakye, D.A., Bockarie, M.J., 2015. The impact of residual infections on Anopheles-transmitted Wuchereria bancrofti after multiple rounds of mass drug administration. Parasit. Vectors 8, 488.

Dewi, R.M., Tuti, S., Ganefa, S., Anwar, C., Larasati, R., Ariyanti, E., Herjati, H., Brady, M., 2015. Brugia Rapid $^{\mathrm{TM}}$ antibody responses in communities of Indonesia in relation to the results of 'transmission assessment surveys' (TAS) for the lymphatic filariasis elimination program. Parasit. Vectors 8, 499. https://doi.org/10.1186/s13071-015-1093-x

Eigege, A., Kal, A., Miri, E., Sallau, A., Umaru, J., Mafuyai, H., Chuwang, Y.S., Danjuma, G., Danboyi, J., Adelamo, S.E., 2013. Long-lasting insecticidal nets are synergistic with mass drug administration for interruption of lymphatic filariasis transmission in Nigeria. PLoS Negl. Trop. Dis. 7.

Kelly-Hope, L.A., Molyneux, D.H., Bockarie, M.J., 2013. Can malaria vector control accelerate the interruption of lymphatic filariasis transmission in Africa; capturing a window of opportunity? Parasit. Vectors 6, 39.

Mahoney, L.E., Kessel, J.F., 1971. Treatment failure in filariasis mass treatment programmes. Bull. World Health Organ. 45, 35.

Meyrowitsch, D.W., Simonsen, P.E., Makunde, W.H., 1995. A 16-year follow-up study on bancroftian filariasis in three communities of north-eastern Tanzania. Ann. Trop. Med. Parasitol. 89, 665-675.

Michael, E., Grenfell, B.T., Bundy, D.A.P., 1994. The association between microfilaraemia and disease in lymphatic filariasis. Proc. R. Soc. London. Ser. B Biol. Sci. 256, 33-40.

Michael, E., Malecela-Lazaro, M.N., Maegga, B.T.A., Fischer, P., Kazura, J.W., 2006. Mathematical models and lymphatic filariasis control: monitoring and evaluating interventions. Trends Parasitol. 22, 529-535.

Mohammed, K.A., Molyneux, D.H., Albonico, M., Rio, F., 2006. Progress towards eliminating lymphatic filariasis in Zanzibar: a model programme. Trends Parasitol. 22, 340-344.

Molyneux, D., 2003. Lymphatic filariasis (elephantiasis) elimination: a public health success and development opportunity. Filaria J. 2, 13. 
Organization, W.H., 2011. Monitoring and epidemiological assessment of mass drug administration in the global programme to eliminate lymphatic filariasis: a manual for national elimination programmes.

Ottesen, E.A., 2000. The global programme to eliminate lymphatic filariasis. Trop. Med. Int. Heal. 5, 591594.

Ottesen, E.A., 1998. The global programme to eliminate lymphatic filariasis [WWW Document]. Parasitol. Int. https://doi.org/10.1016/s1383-5769(98)80076-7

Pion, S.D.S., Chesnais, C.B., Weil, G.J., Fischer, P.U., Missamou, F., Boussinesq, M., 2017. Effect of 3 years of biannual mass drug administration with albendazole on lymphatic filariasis and soil-transmitted helminth infections: a community-based study in Republic of the Congo. Lancet Infect. Dis. 17, 763-769.

Rebollo, M.P., Mohammed, K.A., Thomas, B., Ame, S., Ali, S.M., Cano, J., Escalada, A.G., Bockarie, M.J., 2015a. Cessation of mass drug administration for lymphatic filariasis in Zanzibar in 2006: was transmission interrupted? PLoS Negl. Trop. Dis. 9.

Rebollo, M.P., Sambou, S.M., Thomas, B., Biritwum, N.-K., Jaye, M.C., Kelly-Hope, L., Escalada, A.G., Molyneux, D.H., Bockarie, M.J., 2015b. Elimination of lymphatic filariasis in the Gambia. PLoS Negl. Trop. Dis. 9.

Sheel, M., Sheridan, S., Gass, K., Won, K., Fuimaono, S., Kirk, M., Gonzales, A., Hedtke, S.M., Graves, P.M., Lau, C.L., 2018. Identifying residual transmission of lymphatic filariasis after mass drug administration: Comparing school-based versus community-based surveillance-American Samoa, 2016. PLoS Negl. Trop. Dis. 12, e0006583.

Smits, H.L., 2009. Prospects for the control of neglected tropical diseases by mass drug administration. Expert Rev. Anti. Infect. Ther. 7, 37-56.

Stolk, W.A., Swaminathan, S., Oortmarssen, G.J. van, Das, P.K., Habbema, J.D.F., 2003. Prospects for elimination of bancroftian filariasis by mass drug treatment in Pondicherry, India: a simulation study. J. Infect. Dis. 188, 1371-1381.

WHO, 2010. Progress report 2000-2009 and strategic plan 2010-2020 of the global programme to eliminate lymphatic filariasis: halfway towards eliminating lymphatic filariasis. World Health Organization.

Witt, C., Ottesen, E.A., 2001. Lymphatic filariasis: an infection of childhood. Trop. Med. Int. Heal. 6, 582606.

\section{Figures}




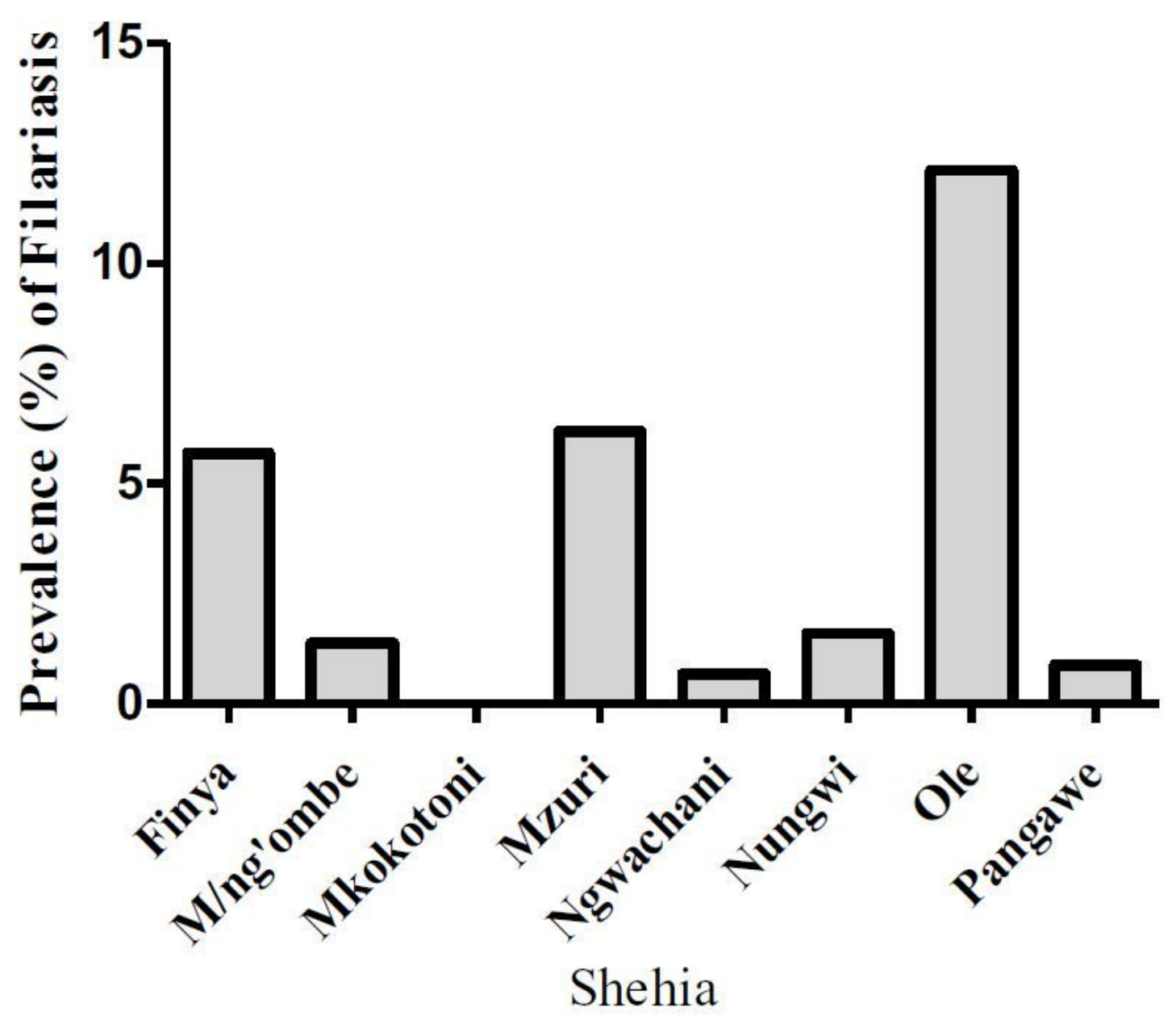

Figure 1

Prevalence of filariasis across different Shehias 


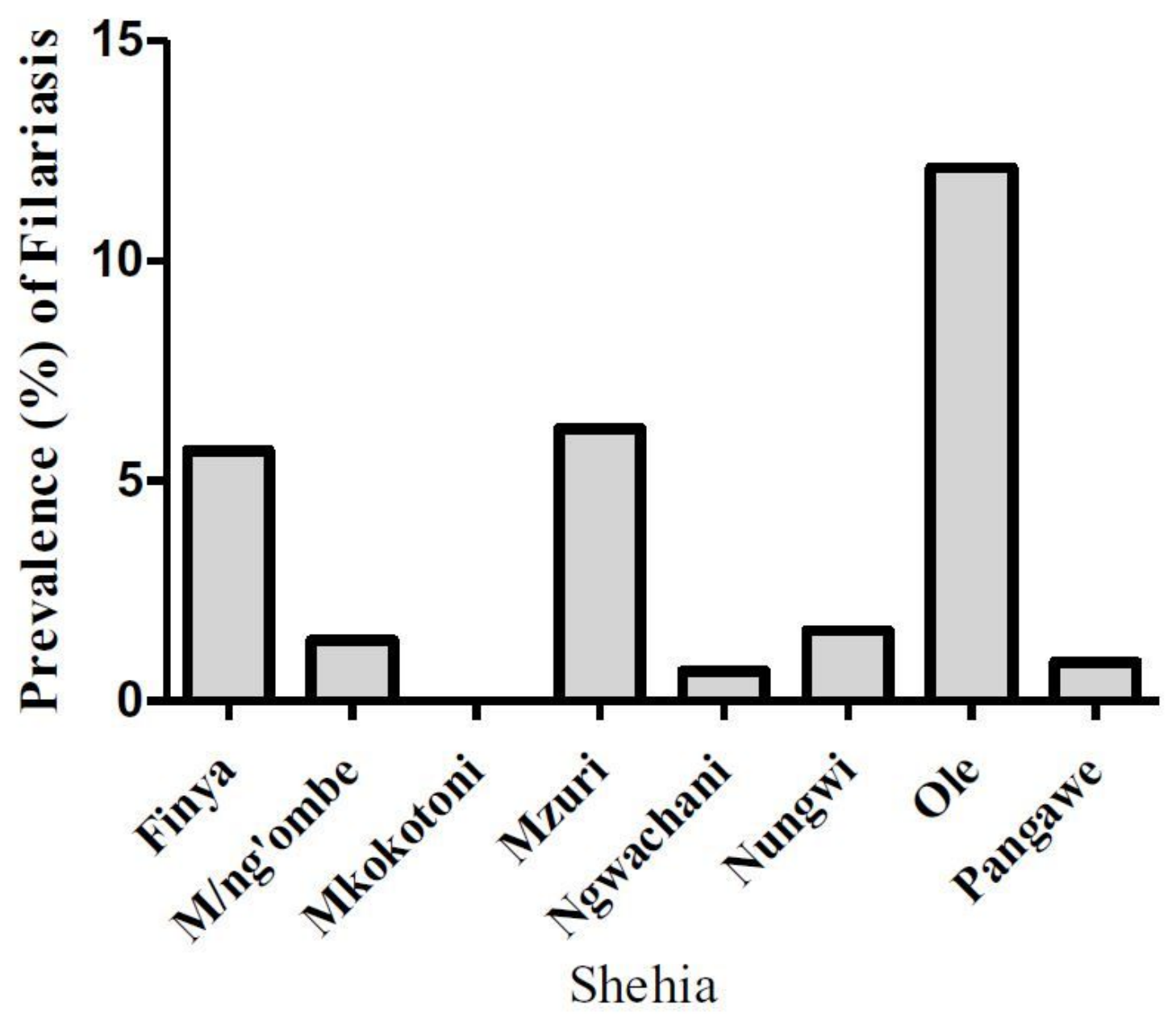

Figure 1

Prevalence of filariasis across different Shehias 


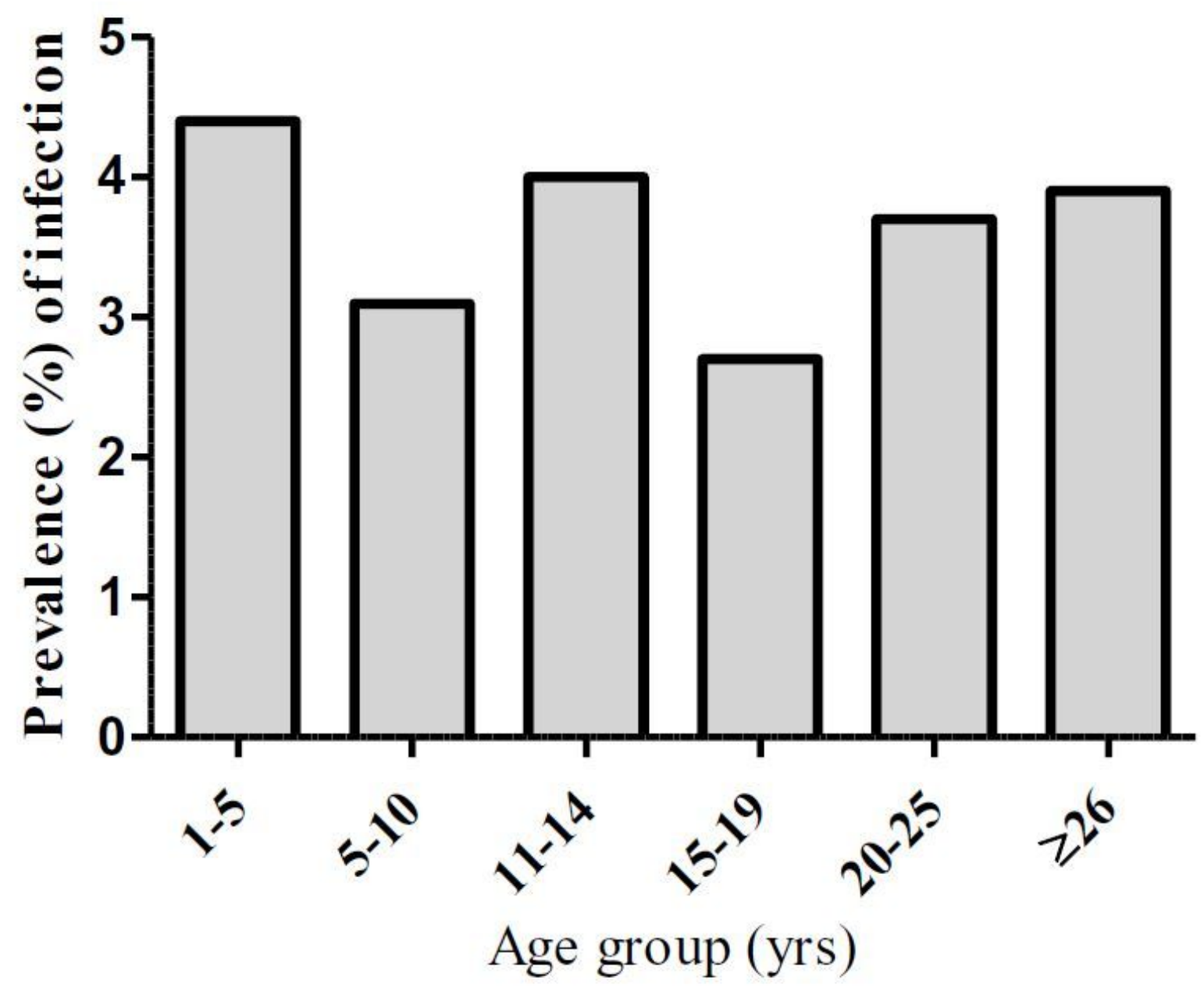

Figure 2

Prevalence of filariasis infection in different age groups in Zanzibar 


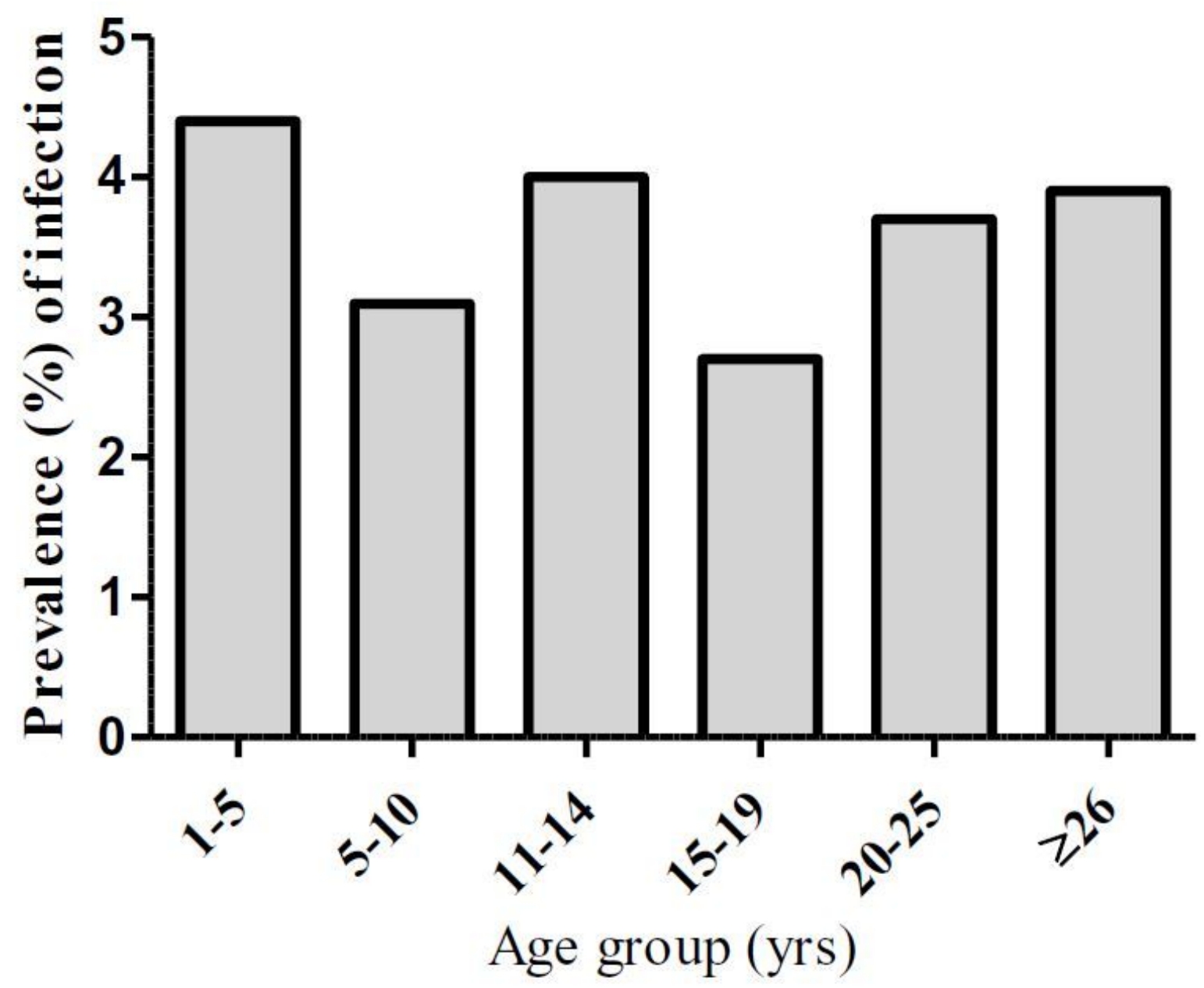

Figure 2

Prevalence of filariasis infection in different age groups in Zanzibar 


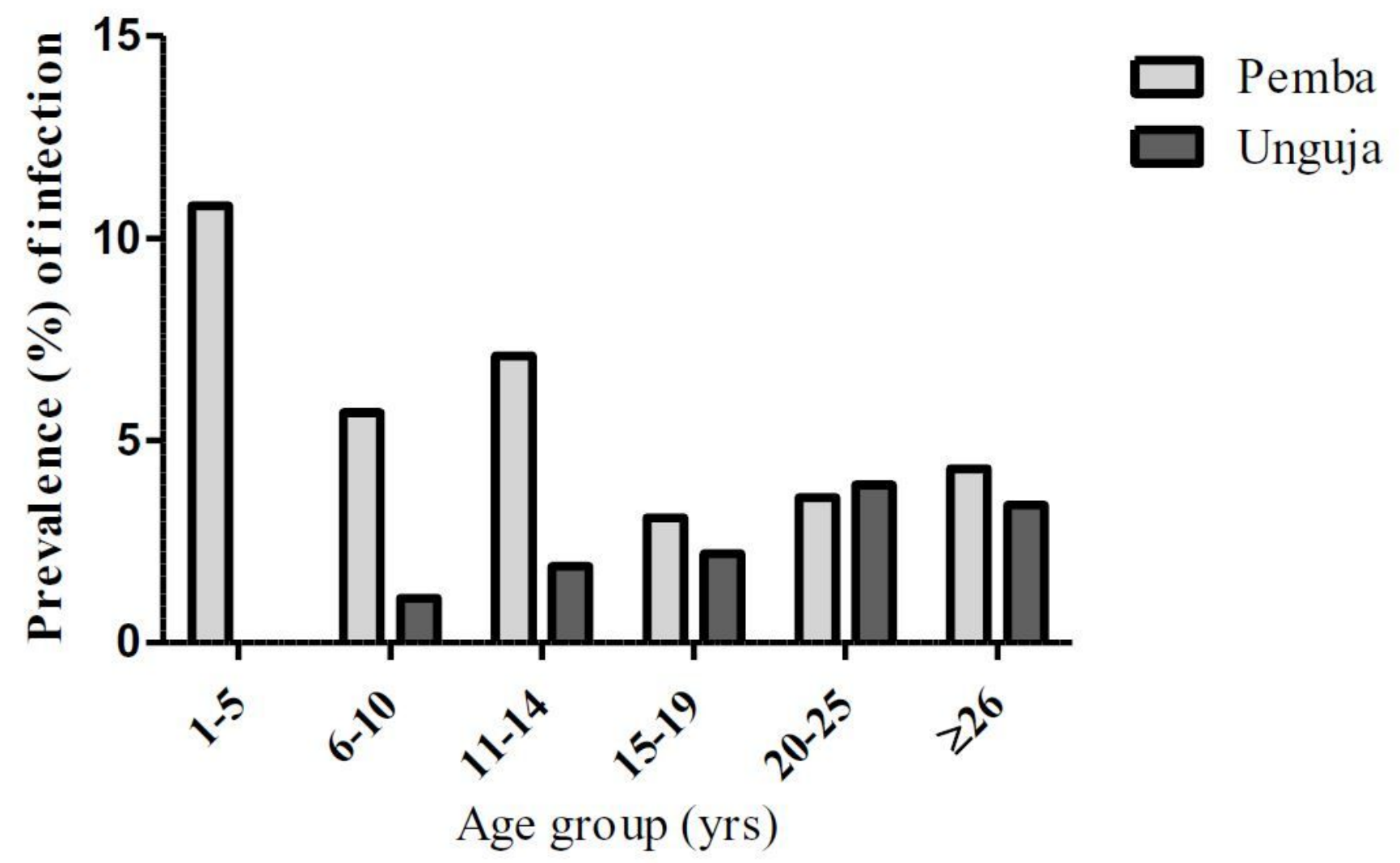

Figure 3

Prevalence of filariasis infection between Pemba and Unguja among different age groups 


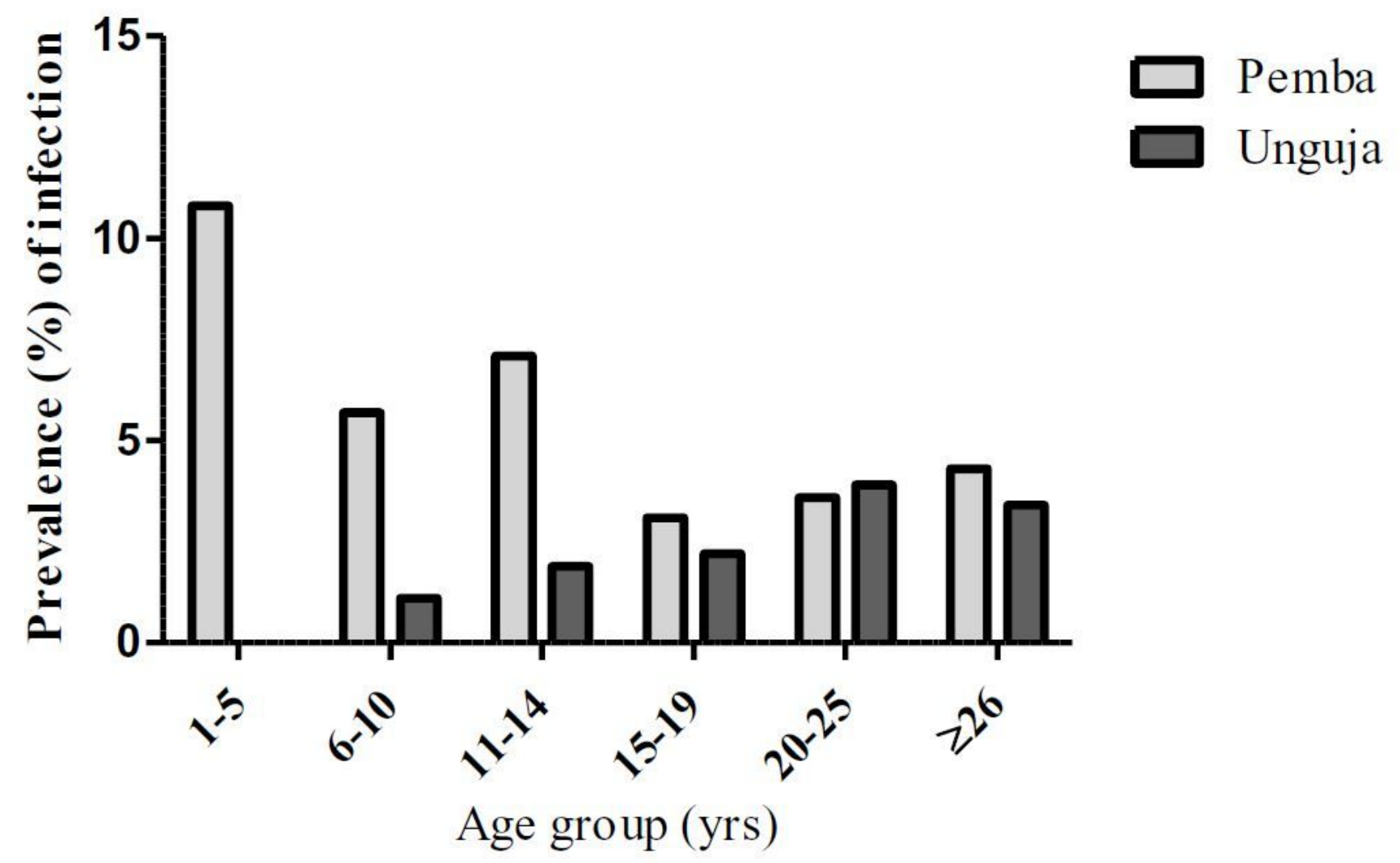

Figure 3

Prevalence of filariasis infection between Pemba and Unguja among different age groups 